\title{
Analysing Soft Clothing Biometrics for Retrieval
}

\author{
Emad Sami Jaha ${ }^{1,2}$, and Mark S. Nixon ${ }^{2}$ \\ Faculty of Computing and Information Technology, King Abdulaziz University, Saudi Arabia ${ }^{1}$ \\ School of Electronics and Computer Science, University of Southampton, United Kingdom ${ }^{2}$
}

\begin{abstract}
Soft biometrics continues to attract research interest. Traditional body and face soft biometrics have been the main research focus and have been proven, by many researchers, to be usable for identification and retrieval. Also, soft biometrics have been shown to provide several advantages over classic biometrics, such as invariance to illumination and contrast. Other than body and face, little attention has focussed on semantic descriptions of an individual, including clothing attributes. Research has yet to concern clothing characteristics as a major or complementary set of biometric traits. In this paper, we analyse the reliability and significance of clothing information for retrieval purposes. We investigate and rate the viability of semantic clothing descriptions to retrieve a subject correctly, given a verbal description of their clothing.
\end{abstract}

Keywords: Soft Biometrics; Human Descriptions; Retrieval; Semantic Clothing Attributes; Relative Attributes

\section{Introduction}

In recent years, there has been an increasing interest in soft biometrics. Traditional soft biometrics such as age, gender, and ethnicity in addition to body and face traits like height, and arm length, have been the most considered traits for different objectives and in a variety of applications.

Subject retrieval is a useful and challenging biometric application. Bodily human features can be described using human understandable labels and measurements, which in turn, allow for recognition and retrieval using only verbal descriptions as the sole query $[1,2]$. The features also allow prediction of other measurements as they have been observed to be correlated [3]. Indeed, soft traits are not unique to an individual but a discriminative biometric signature can be designed from their aggregation. Verbal identification can be used to retrieve subjects who have been previously enrolled in database [4] and it could be extended, in a more challenging application, for retrieval from video footage [1]. The capability of verbal retrieval from images and videos can pave the way for applications that can search surveillance data of a crime scene to match people to potential suspects described verbally by eyewitnesses. Soft biometric databases based on categorical labels can be incorporated with other biometrics to enhance recognition, such as integrating soft body traits with a gait signature [4], and using soft facial traits along with other (hard) facial traits [5]. Nevertheless, soft 
comparative labels have been demonstrated to be more successful in representing the slight differences between people in bodily descriptions [1]. Facial marks, for instance, can be automatically detected and ascribed to be used as micro soft traits to supplement primary facial features for improved face recognition and fast retrieval, besides they may enable matching with low resolution or partial images [6,7]. For surveillance purposes, different forms of soft biometrics take place in various means of applications and scenarios $[1,8,9]$.

Human clothes are a predominant visible characteristic of the person's appearance. However, clothing has rarely been adopted for representing soft biometric traits for an individual and has been considered unlikely to be a clue to identity [10]. Clothing can reflect some cues regarding social status, lifestyle and cultural affiliation. In addition, clothing encodes more information about an individual, beyond just their visual appearance [10]. There are few research studies associated with using clothing for biometric purposes $[2,5,9,11,12]$. The majority of existing research employs computer vision algorithms and machine learning techniques to extract and use visual clothing descriptions in applications including: online person recognition $[5,11]$; semantic attributes for re-identification [13]; detecting and analysing semantic descriptions (labels) of clothing colours and types to supplement other bodily and facial soft attributes in automatic search and retrieval [9]; and utilizing some clothing attributes like colour [14] and style to improve the observation and retrieval at a distance in surveillance environments [2]. Even with images captured on different days, there remains sufficient information to compare and establish identity, since clothes are often re-worn or a particular individual may prefers a specific clothing style or colour [15]. Clothing descriptions like indicative colours and decorations could be utilized to supplement other behavioural biometrics like human motion pattern, hence they can form a biometric fingerprint that serves as a person's identifier [11].

This research aims to investigate the capability of soft clothing traits towards reinforcing biometric signatures. We have previously studied the identification capability of these new measures [16]. This paper focusses on using clothing to enable accurate subject retrieval, and the efficacy of the clothing labels. Furthermore, a set of experiments validates and evaluates the retrieval performance of clothing-based techniques and the new sets of clothing labels. We outline and discuss their retrieval performance, measured by a set of evaluation metrics. The main contributions of this paper comprise:

- extended analysis and investigation of reliability and significance of proposed categorical and comparative soft clothing traits;

- new soft clothing-based biometric techniques for subject retrieval; and

- detailed retrieval assessment and comparison of soft clothing approaches.

Section 2 outlines the proposed semantic attributes and their labels. Section 3 explains the mechanism used for data collection and clothing database design. Section 4 introduces soft clothing biometrics. Section 5 demonstrates clothing information analysis. Section 6 describes subject retrieval using soft clothing biometrics. Finally, Section 7 concludes the paper and discusses future work. 
Table 1. Semantic clothing attributes and corresponding categorical and comparative labels

\begin{tabular}{|c|c|c|c|}
\hline Body zone & Semantic Attribute & Categorical Labels & Comparative Labels \\
\hline \multirow{4}{*}{ Head } & 1. Head clothing category & [None, Hat, Scarf, Mask, Cap] & \\
\hline & 2. Head coverage & [None, Slight, Fair, Most, All] & $\begin{array}{l}\text { [Much Less, Less, Same, } \\
\text { More, Much more] }\end{array}$ \\
\hline & 3. Face covered & [Yes, No, Don't know] & $\begin{array}{l}\text { [Much Less, Less, Same, } \\
\text { More, Much more] }\end{array}$ \\
\hline & 4. Hat & [Yes, No, Don't know] & \\
\hline \multirow{4}{*}{$\begin{array}{l}\text { Upper } \\
\text { body }\end{array}$} & 5. Upper body clothing category & $\begin{array}{l}\text { [Jacket, Jumper, T-shirt, Shirt, Blouse, } \\
\text { Sweater, Coat, Other] }\end{array}$ & \\
\hline & 6. Neckline shape & $\begin{array}{l}\text { [Strapless, V-shape, Round, Shirt collar, } \\
\text { Don't know] }\end{array}$ & \\
\hline & 7. Neckline size & $\begin{array}{l}\text { [Very Small, Small, Medium, Large, } \\
\text { Very Large] }\end{array}$ & $\begin{array}{l}\text { [Much Smaller, Smaller, } \\
\text { Same, Larger, Much Larger] }\end{array}$ \\
\hline & 8. Sleeve length & $\begin{array}{l}\text { [Very Short, Short, Medium, Long, } \\
\text { Very Long] }\end{array}$ & $\begin{array}{l}\text { [Much Shorter, Shorter, } \\
\text { Same, Longer, Much Longer] }\end{array}$ \\
\hline \multirow{4}{*}{$\begin{array}{l}\text { Lower } \\
\text { body }\end{array}$} & 9. Lower body clothing category & [Trouser, Skirt, Dress] & \\
\hline & 10. Shape & [Straight, Skinny, Wide, Tight, Loose] & \\
\hline & 11. Leg length (of lower clothing) & $\begin{array}{l}\text { [Very Short, Short, Medium, Long, } \\
\text { Very Long] }\end{array}$ & $\begin{array}{l}\text { [Much Shorter, Shorter, } \\
\text { Same, Longer, Much Longer] }\end{array}$ \\
\hline & 12. Belt presence & [Yes, No, Don't know] & \\
\hline \multirow[b]{2}{*}{ Foot } & 13. Shoes category & [Heels, Flip flops, Boot, Trainer, Shoe] & \\
\hline & 14. Heel level & [Flat/low, Medium, High, Very high] & $\begin{array}{l}\text { [Much Lower, Lower, Same, } \\
\text { Higher, Much higher] }\end{array}$ \\
\hline \multirow{5}{*}{$\begin{array}{l}\text { Attached } \\
\text { to body }\end{array}$} & 15. Attached object category & [None, Bag, Gun, Object in hand, gloves] & \\
\hline & 16. Bag (size) & $\begin{array}{l}\text { [None, Side-bag, Cross-bag, Handbag, } \\
\text { Backpack, Satchel] }\end{array}$ & $\begin{array}{l}\text { [Much Smaller, Smaller, } \\
\text { Same, Larger, Much Larger] }\end{array}$ \\
\hline & 17. Gun & [Yes, No, Don't know] & \\
\hline & 18. Object in hand & [Yes, No, Don't know] & \\
\hline & 19. Gloves & [Yes, No, Don't know] & \\
\hline $\begin{array}{l}\text { General } \\
\text { style }\end{array}$ & 20. Style category & $\begin{array}{l}\text { [Well-dressed, Business, Sporty, } \\
\text { Fashionable, Casual, Nerd, Bibes, Hippy, } \\
\text { Religious, Gangsta, Tramp, Other] }\end{array}$ & \\
\hline Permanent & 21. Tattoos & [Yes, No, Don't know] & \\
\hline
\end{tabular}

\section{Semantic Clothing Attributes}

A subject's clothing can be described using different semantic attributes. For the purpose of this research, amongst several possible clothing attributes and labels, an initial set of attributes is considered (see Table 1, as described elsewhere [16]). A group of categorical and comparative labels are used to describe these attributes.

Categorical labels can be defined as nameable descriptions used to describe semantic attributes of an individual's clothing, usually associated with multiple clothing categories or styles such as (Upper body clothing category: 'Jacket', 'Jumper', 'T-shirt' etc.) or can be labels describing the degree of presence of relative attributes such as (Sleeve length: 'Very short', 'Short', 'Medium' etc.). Comparative labels are nameable descriptions used to describe only relative attributes of an individual's clothing compared with another individual's clothing. In other words, these labels describe the degree of comparisons of relative attributes, such as (Neckline size: 'Much smaller', 'Smaller', 'Same', 'Larger' and 'Much larger').

A list of 21 semantic attributes is proposed and each attribute is described by a specified group of suitable categorical labels. Furthermore, seven of the 21 attributes are both categorical and relative enabling comparison, whereas the remaining 14 are 
unsuited for comparison because they are binary or multi-class attributes that can be described using only categorical (absolute) labels. In Table 1 the seven relative comparable attributes are in bold. We utilise an early analysis [17] used bipolar scales to define traits for whole-body descriptions. For all binary-label clothing attributes, such as 'Belt presence', a label 'Don't know' was included as a choice.

\section{Data Acquisition}

The Soton Gait Database [18] is a standard database and comprises a subset of fullbody fronta and side view still images. The front view images are used to collect clothing descriptions. This subset consists of 115 individuals with a total of 128 front view samples. Here, each sample is handled as an independent individual; multiple samples of a single individual are considered as different and independent entities by which each entity represents that individual only if wearing exactly the same clothing. Otherwise, it is considered as another entity, even though it belongs to the same individual. Here, each entity (i.e. sample in this dataset) is referred to as a subject. Note that $90 \%$ of the subjects in the database are university students and wear largely similar (summer) clothing (jeans, T-shirt, etc.) and the data appears sufficiently challenging for this initial study.

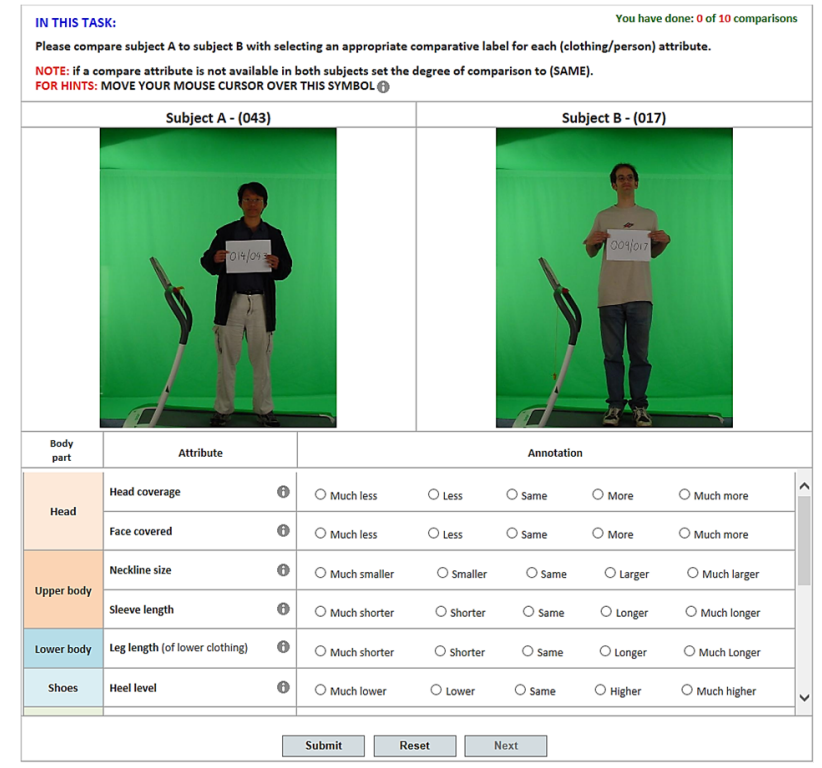

Fig. 1. Comparative Form of the website developed to obtain annotation data

A website, shown in Fig. 1, was designed and developed to obtain clothing labels and comparisons, through two tasks. The first task required a user to annotate ten subjects. Each subject was described by selecting 21 appropriate categorical labels. The second task required a user to compare one subject, selected randomly from the ten already annotated, with other ten new subjects. A comparison, between two subjects 
was performed by selecting seven appropriate comparative labels. In this way 27 labellers provided a total of 12747 categorical and comparative labels on 128 subjects. Clothing attributes were grouped based on their zones and relevance as: Head, Upper body, Lower body, Foot, Attached to body, General style and Permanent as shown in Table 1 and Fig. 1.

6636 categorical and 2219 comparative labels were collected from the 27 users via the website. All 128 samples were labelled by multiple users, with one or more separate user annotations per subject describing the 21 categorical attributes such that, each subject's annotation provided by a single annotator. All subjects were compared using the seven relative attributes by multiple users. To enrich the comparison data from the available number of collected comparisons, 3892 additional comparisons were inferred when two subjects were both compared with another same subject.

\section{Soft Clothing Biometrics}

\subsection{Categorical Clothing Traits ( $C a t-N)$}

Categorical annotations are used to form a categorical-based feature vector for each subject in a Training dataset. This feature vector is deduced by computing a normalized average-label per attribute for a set of labels provided by multiple users describing the same subject. The resulting 21 attribute values per subject are used to form a number of categorical feature vectors and to construct their galleries, containing the same type of feature vectors for all subjects in database. The first feature vector is formed using the values of all the 21 attributes, constructing a gallery called Cat-21. The second feature vector is formed from the values of a subset of the only seven relative clothing attributes, shown in bold in Table 1 (attributes 2, 3, 7, 8, 11, 14, and 16), to build a gallery called Cat-7.

Feature Subset Selection. A third feature vector is formed by applying one-way analysis of variance (ANOVA) to determine the most effective traits (and attributes) for discrimination. After analysing traits separately, traits were ranked as shown in Fig. 2. A minimum number of traits that achieve the best retrieval performance were selected. The third feature vector is formed using a subset of the top five traits (attributes 2, 8, 9, 12, and 11) outlined in Table 2-(a), building a gallery called (Cat-5).

\subsection{Comparative Clothing Traits ( $\mathrm{Cmp})$}

Comparison data can be used to convey meaningful information describing a subject in relation to the remaining population [1]. The collected comparative annotations need to be anchored, per attribute, to define invariant relative measurements for each subject. To derive these measurements, a ranking method needs to be applied to arrange a list of ordered subjects with respect to a single attribute. In the ranking process, the comparisons between subjects are used as rules to enforce ordering for subjects, and to adjust a relative measurement per attribute for each. 
Ranking SVM. To achieve ordering and to derive the desired relative measurements to represent comparative soft clothing traits, a soft-margin Ranking SVM method [19] is used, along with a supporting formulation of similarity constraints [20]. This applies a pairwise technique based on learning a ranking function per attribute, which can be used not only to determine the relative strength of attributes in a training sample, but also to predict the relative strength in a new test sample. Thus, for a set of attributes $A$, a ranking linear function $r_{a}$ is learned for each attribute $a$ such that:

$$
r_{a}\left(x_{i}\right)=w_{a}^{T} x_{i}
$$

where $w_{a}$ is the coefficient of the ranking function $r_{a}$ and $x_{i}$ is a feature vector of attributes of a subject being ranked. A set of comparisons is rearranged into two groups to represent the pairwise relative constraints required to learn a ranking function. The first group consists of a set of dissimilarity comparisons $D_{a}$ of ordered pairs so that $(i$, $j) \in D_{a} \Rightarrow i>j$ whereas the second group comprises a set of similarity comparisons $S_{a}$ of non-ordered pairs so that $(i, j) \in S_{a} \Rightarrow i=j . D_{a}$ and $S_{a}$ sets are then utilized to derive the $w_{a}$ coefficients of $r_{a}$ according to the following formulation:

$$
\begin{aligned}
\operatorname{minimise} & \left(\frac{1}{2}\left\|w_{a}^{T}\right\|^{2}+C \sum \xi_{i j}^{2}\right) & \\
\text { subject to } & w_{a}^{T}\left(x_{i}-x_{j}\right) \geq 1-\xi_{i j} ; & \forall(i, j) \in D_{a} \\
& \left|w_{a}^{T}\left(x_{i}-x_{j}\right)\right| \leq \xi_{i j} ; & \forall(i, j) \in S_{a} \\
& \xi_{i j} \geq 0 &
\end{aligned}
$$

The degree of misclassification is measured by $\xi_{i j}$ and the trade-off between maximizing the margin and minimizing the error (i.e. satisfying constraints) is denoted as $C$. The resulting optimal function $w_{a}$ can enforce (explicitly) a desirable ordering for all training samples, in respect to $a$. A feature vector $x_{i}$ is mapped using Eqn. (1) to a corresponding feature vector comprising a number of real-value relative measurements. Each measurement represents the relative strength of a single attribute.

All subjects in the Cat-7 gallery are used as a training dataset to learn seven optimal ranking functions for the seven relative attributes. The weighting of each function is derived using the formulation in Eqn. (2). The desirable per attribute ordering of all subjects is deduced from $w$. Then by Eqn. (1), each value of $w$ is used to map each feature vector in $\mathrm{Cat}-7$ to a corresponding vector of seven relative measurements (i.e. comparative traits) describing a single subject. All the obtained relative measurement vectors are gathered to compose a fourth gallery called $(\mathrm{Cmp})$.

\section{Data Analysis}

\subsection{Analysis Of Variance (ANOVA)}

Table 2 provides the ordered lists of resulting ANOVA test values for categorical and comparative clothing traits. Accordingly, Fig. 2 shows ordered p-values scaled positively by computing the absolute logarithm of the p-value, which emphasises 
smaller p-values. Scaling is used to magnify small differences between $p$-values and to be visually observable. Head coverage is highly discriminative since few subjects had covered heads. It is perhaps surprising that sleeve length is so discriminative, especially compared with the length of the trousers, but that is what this analysis reveals, and no summary analysis is possible by human vision.

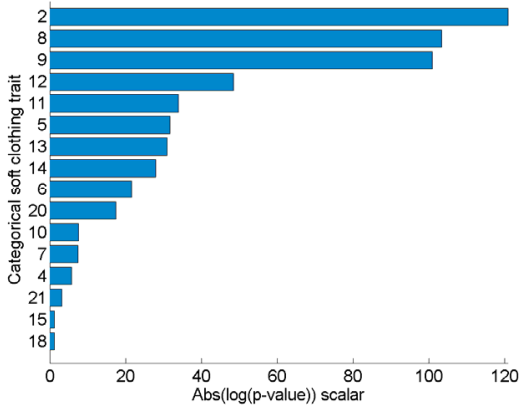

(a) Categorical traits

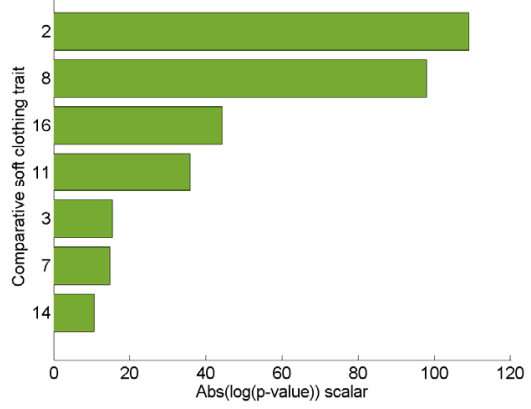

(b) Comparative traits

Fig. 2. Attributes ordered according to $p$-values

Table 2. Ordered list of clothing traits by their F-ratios

(a) Categorical trait

\begin{tabular}{|l|l|l|}
\hline \multicolumn{1}{|c|}{ Soft clothing biometrics } & $\begin{array}{c}\text { F-ratio } \\
(d f=315)\end{array}$ & $\begin{array}{c}\text { P-value } \\
(\mathrm{p} \leq 0.05)\end{array}$ \\
\hline 2. Head coverage & 13.239 & $8.45 \mathrm{E}-53$ \\
\hline 8. Sleeve length & 10.549 & $3.07 \mathrm{E}-45$ \\
\hline 9. Lower body clothing category & 10.189 & $4.07 \mathrm{E}-44$ \\
\hline 12. Belt presence & 4.600 & $2.46 \mathrm{E}-21$ \\
\hline 11. Leg length (of lower clothing) & 3.478 & $5.07 \mathrm{E}-15$ \\
\hline 5. Upper body clothing category & 3.324 & $4.25 \mathrm{E}-14$ \\
\hline 13. Shoes category & 3.265 & $9.66 \mathrm{E}-14$ \\
\hline 14. Heel level & 3.057 & $1.80 \mathrm{E}-12$ \\
\hline 6. Neckline shape & 2.608 & $1.16 \mathrm{E}-09$ \\
\hline 20. Style category & 2.326 & $6.96 \mathrm{E}-08$ \\
\hline 10. Shape (of lower clothing) & 1.618 & 0.0013 \\
\hline 7. Neckline size & 1.608 & 0.0015 \\
\hline 4. Hat & 1.471 & 0.0081 \\
\hline 21. Tattoos & 1.214 & 0.1129 \\
\hline 15. Attached object category & 0.874 & 0.7910 \\
\hline 18. Object in hand & 0.874 & 0.7910 \\
\hline
\end{tabular}

(b) Comparative trait

\begin{tabular}{|l|l|l|}
\hline \multicolumn{1}{|c|}{$\begin{array}{c}\text { Soft clothing } \\
\text { biometrics }\end{array}$} & $\begin{array}{c}\text { F-ratio } \\
(d f=315)\end{array}$ & $\begin{array}{c}\text { P-value } \\
(\mathrm{p} \leq 0.05)\end{array}$ \\
\hline 2. Head coverage & 11.369 & $1.08 \mathrm{E}-47$ \\
\hline 8. Sleeve length & 9.790 & $7.79 \mathrm{E}-43$ \\
\hline 16. Bag (size) & 4.258 & $1.73 \mathrm{E}-19$ \\
\hline $\begin{array}{l}\text { 11. Leg length } \\
\text { (of lower clothing) }\end{array}$ & 3.622 & $7.16 \mathrm{E}-16$ \\
\hline 3. Face covered & 2.186 & $5.30 \mathrm{E}-07$ \\
\hline 7. Neckline size & 2.140 & $1.03 \mathrm{E}-06$ \\
\hline 14. Heel level & 1.852 & $6.07 \mathrm{E}-05$ \\
\hline
\end{tabular}

\subsection{Correlations and Significance}

The exploration of clothing traits' significance and correlations, is deemed to be an important analysis resulting in a better comprehension of which of traits contribute most to identification and leading to wider potential predictability of other traits [3]. The proposed clothing traits were assessed to investigate their correlation and effectiveness for subject description. For the sake of this investigation, we used the correlation matrix computed using (Pearson's $r$ ) correlation coefficient to highlight the significance of an 
attribute and the mutual relations between traits. Note that, when one or both of correlated labels are binary or multi-class describing pure (nominal) categorical traits, this indicates that they are simultaneously present in a single annotation. That is because the assigned numeral values of such labels are unlike (ordinal) labels of relative measurements, so they can be assigned values in any order or their values can be exchanged.

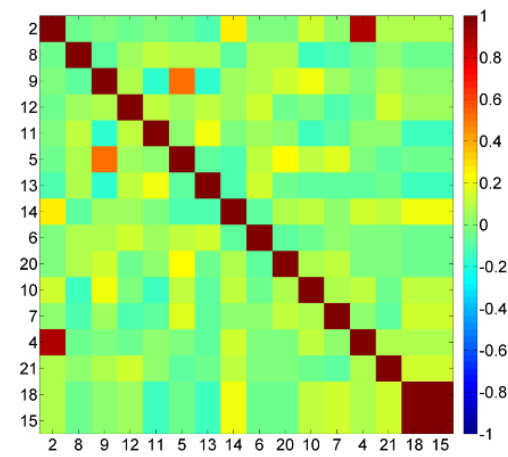

(a) Categorical clothing traits

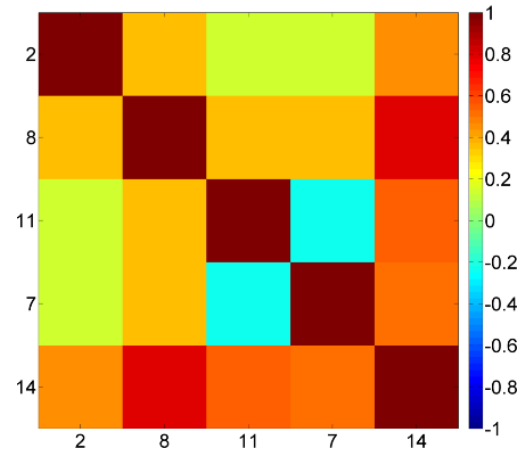

(b) Comparative clothing traits

Fig. 3. Correlation matrix between the soft clothing traits

Fig. 3 demonstrates the correlation between the most significant categorical traits and comparative traits (see Table 2); traits without correlation are not shown. High correlation is symbolized by orange, and low by blue/green. In the categorical matrix, traits relating to head coverage (2) and (4) are highly correlated, as are the traits (15) and (18) relating to the description of items attached to the body. Clothing categories are well correlated for upper (5) and lower (9) body, as expected. In the comparative matrix, sleeve length (8) and heel level (14) are highly correlated. Heel level is also well correlated with leg length (11) and neckline size (7). The structures of both correlation matrices suggest that the desired uniqueness has indeed been achieved.

\section{$6 \quad$ Retrieval using Soft Clothing Traits}

The main objective of this experimental work is to validate and evaluate the proposed soft clothing approaches, described in Section 4, in retrieval and to explore their viability to supplement the performance of soft body biometrics. The distinction between retrieval and recognition concerns the ability to generalise to unseen data. We use the previously collected soft body descriptions from the Soton database [11] where each of 115 individuals was labelled by multiple users' describing 23 soft bodily traits. These traits were grouped into three categories: Body shape, Global, and Head. Our clothing analysis is used to enhance the retrieval in two different respects. Firstly, to enhance the performance of only the traditional soft traits (Age, Ethnicity, Sex, and Skin Colour) which were grouped as Global attributes as in [4]. Secondly, to enhance all the mentioned 23 soft body traits including the four traditional traits.

Biometric based retrieval can be described as a task that aims to identify an unknown 
subject by comparing and matching their biometric signature with those signatures enrolled in a database [1]. For the sake of retrieval, the collected clothing annotations were divided into two sets: a Query set comprising one annotation per subject for each of the 128 subjects, which is used (as unseen data) to examine retrieval; and a Training set containing all the remaining annotations, which is used for training and feature selection processes. All annotations in the Query set are excluded from the label data, and not reused in Training set and not involved in any training processes. The body Training set is used to derive for each subject a single feature vector consisting of 23 normalized average-labels. These average-labels are computed for a set of annotations provided by multiple users describing the 23 body traits of the same subject. A set of all computed feature vectors comprises a gallery called softBody to be tested separately in retrieval. Then each of the soft clothing galleries (Cat-21, Cat-7, Cat-5, and Cmp) is used to supplement softBody such that, each feature vector describing a single subject in softBody is concatenated to a corresponding feature vector describing the same subject in each of clothing galleries, resulting in: softCat-21, softCat-7, softCat-5 and softCmp galleries. Another gallery called tradSoft is derived from softBody, consisting of a four-trait feature vector per subject subset of only comprising only the four traditional soft descriptions (i.e. Age, Ethnicity, Sex, and Skin Colour). Likewise, tradSoft is extended to four versions supplemented by clothing to construct new galleries: tradCat-21, tradCat-7, tradCat-5 and tradCmp. Query-vectors are normalised and reshaped according to the feature-vectors in a tested gallery to enable comparison and matching.

The likelihood between every single query-vector and all subject-vectors in a gallery is estimated and retrieved, resulting in an ordered list of all subjects based on likelihood evaluated by the sum of Euclidean distance between query and gallery vectors. A number of standard performance evaluation methods are used to enable comparison between approaches from different perspectives. The Cumulative Match Characteristic (CMC) curve is applied to summarize the retrieval accuracy, which scores the existence of the correct subjects within a (likelihood-based) ranked list starting from list-length of 1 to 128 the total number of subjects in a tested gallery. Receiver Operator Characteristic (ROC) analysis is used to assess and compare the approaches performance and their generated errors. A set of further performance metrics are deduced from the ROC analysis comprising the Area Under the Curve (AUC) as in our consideration a smaller are under the ROC curve reflecting a less error and a better performance, and the Equal Error Rate (EER). Also the Decidability Index $\left(d^{\prime}\right)$ metric is computed from the normalized distance between the two means of Genuine $(G)$ and Imposter (I) distributions such that $d^{\prime}=\left|\mu_{G}-\mu_{I}\right| / \sqrt{\left(\sigma_{G}^{2}+\sigma_{I}^{2}\right) / 2}$. The overall performance, with respect to all evaluation metrics, is deduced for all approaches to rank them by overall score.

\subsection{Retrieval using Clothing and Traditional Soft Biometrics}

The ROC performance of the examined approaches is compared in Fig. 4, where all clothing approaches but tradCat-21 provide better retrieval accuracy and less error. Table 3 reports the CMC scores and the average-sum scores along different ranks, 
besides the ROC analysis results for the traditional soft traits (Age, Ethnicity, Sex, and Skin Colour) and when adding soft clothing traits to them; all the best values are shown in bold. In all the retrieval match score, all clothing approaches enhance the retrieval performance of the traditional soft biometrics up to $9 \%$ in average at rank 128 achieved by tradCat-5. The best overall performance achieved by tradCat-5 followed by tradCmp with slightly low performance and very close scores across all assessment metrics. However, tradCmp receives the best scores in the average score up to rank 10 and reaching, and tradCat-7 attains the highest score at rank 1, then is exceeded by tradCat-5 and tradCmp with the rank increase.

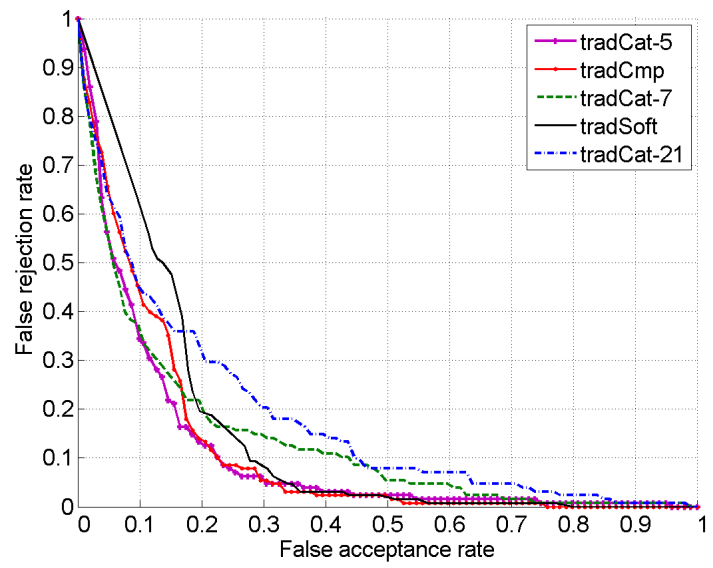

Fig. 4. ROC performance of traditional soft biometrics and when supplemented by clothing

Table 3. Performance metrics of traditional soft biometrics and when supplemented by clothing

\begin{tabular}{|l|c|c|c|c||c|c|c|c|}
\hline \multirow{2}{*}{ Approach } & $\begin{array}{c}\text { Top } \\
\text { rank }\end{array}$ & \multicolumn{2}{|c|}{$\begin{array}{c}\text { AVG sum match } \\
\text { scores up to rank }\end{array}$} & $\begin{array}{c}\text { 100\% accuracy } \\
\text { achieved at rank }\end{array}$ & EER & \multirow{2}{*}{ AUC } & \multirow{2}{*}{$\boldsymbol{d}^{\prime}$} & $\begin{array}{c}\text { Performance } \\
\text { overall rank }\end{array}$ \\
\cline { 2 - 5 } & $=\mathbf{1}$ & $=\mathbf{1 0}$ & $=\mathbf{1 2 8}$ & & & & & \\
\hline tradSoft & 0.14 & 0.27 & 0.838 & 106 & 0.094 & 0.039 & 2.379 & 4 \\
\hline tradCat-21 & 0.23 & 0.41 & 0.866 & 108 & 0.107 & 0.050 & 1.829 & 5 \\
\hline tradCat -7 & $\mathbf{0 . 2 5}$ & 0.47 & 0.900 & 95 & 0.116 & 0.039 & 2.292 & 3 \\
\hline tradCat 5 & 0.24 & 0.49 & $\mathbf{0 . 9 2 5}$ & 70 & $\mathbf{0 . 0 8 6}$ & $\mathbf{0 . 0 3 2}$ & $\mathbf{2 . 4 3 6}$ & $\mathbf{1}$ \\
\hline tradCmp & 0.24 & $\mathbf{0 . 5 0}$ & 0.919 & $\mathbf{6 7}$ & 0.094 & 0.037 & 2.416 & 2 \\
\hline
\end{tabular}

\subsection{Retrieval using Clothing and soft body biometrics}

Fig. 5 presents the CMC curves of the retrieval performance of soft body traits and compare the performance the clothing approaches. The figure shows the CMC curves up to rank 25 where the differences between compared approaches are more significant and can be appreciated. Table 4 provides all the produced metric results of CMC and ROC of body soft biometrics and the clothing approaches. The approaches softCat-5, softCmp, and softCat-7 respectively gain a highest performance that improves retrieval performance of using soft body biometrics alone, softCat-21 starts with a higher accuracy than softBody but provides a lower performance between rank 2 and 23, and then increase rapidly over all approaches. 
The best overall performance is achieved by softCat-5 as it yields the best scores in all evaluation measurements but two as can be observed in Table 4. All clothing approaches started with better retrieval accuracy at rank 1 than the soft body traits, while softCat-5 considerably increase the retrieval from only $67 \%$ to $82 \%$. Although the inferiority of the softCat-21 in all used evaluation criteria compared with its clothing-based counterparts, it is the first to reach the reach $100 \%$ at a minimum rank of 32. softCmp receives the best score in terms of decidability metric $d^{\prime}$.

Table 4. Performance metrics of body soft biometrics and when supplementd by clothing

\begin{tabular}{|c|c|c|c|c|c|c|c|c|}
\hline \multirow[t]{2}{*}{ Approach } & \multirow{2}{*}{$\begin{array}{l}\text { Top } \\
\text { rank } \\
=1\end{array}$} & \multicolumn{2}{|c|}{$\begin{array}{l}\text { AVG sum match } \\
\text { scores up to rank }\end{array}$} & \multirow{2}{*}{$\begin{array}{c}100 \% \text { accuracy } \\
\text { achieved at rank }\end{array}$} & \multirow[t]{2}{*}{ EER } & \multirow[t]{2}{*}{ AUC } & \multirow[t]{2}{*}{$d^{\prime}$} & \multirow{2}{*}{$\begin{array}{l}\text { Performance } \\
\text { overall rank }\end{array}$} \\
\hline & & $=10$ & $=128$ & & & & & \\
\hline softBody & 0.668 & 0.900 & 0.988 & 56 & 0.196 & 0.146 & 1.611 & 4 \\
\hline softCat-21 & 0.695 & 0.882 & 0.987 & 32 & 0.259 & 0.177 & 0.942 & 5 \\
\hline softCat-7 & 0.742 & 0.923 & 0.990 & 49 & 0.209 & 0.133 & 1.417 & 3 \\
\hline softCat-5 & 0.820 & 0.946 & 0.992 & 47 & 0.167 & 0.105 & 1.552 & 1 \\
\hline soft $\mathrm{Cmp}$ & 0.742 & 0.927 & 0.990 & 40 & 0.170 & 0.113 & 1.770 & 2 \\
\hline
\end{tabular}

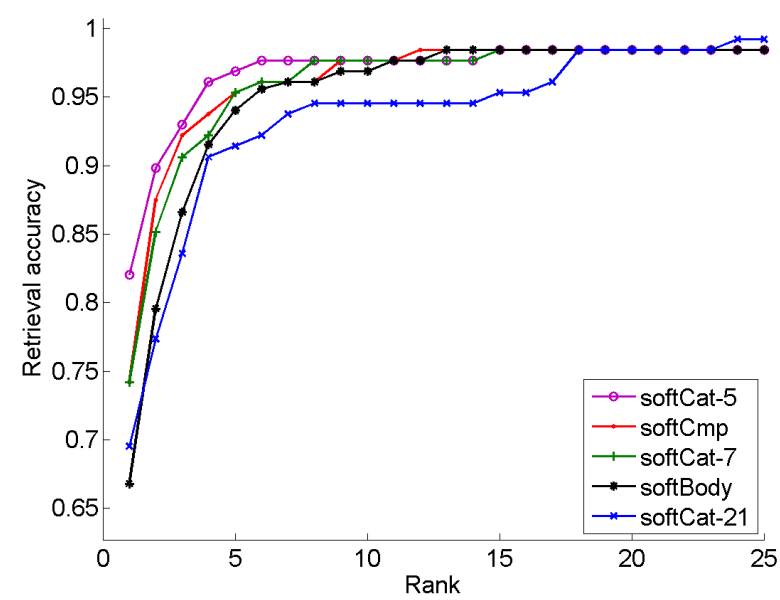

Fig. 5. CMC performance (up to rank 25) of the soft body biometrics and when supplementd by clothing

\section{Conclusions and Future Work}

This paper explores the viability of using soft clothing attributes to achieve enhanced subject retrieval. The results of this exploration using clothing traits highlights a potentially valuable addition to the field of soft biometrics. This can lead to new and useful enhanced biometric applications and systems, using soft clothing biometrics for various purposes including subject search, retrieval, identification, and reidentification. Our analysis of soft clothing traits indicates that such clothing characteristics can be associated in biometric signatures and achieve successful subject retrieval.

Future work will continue to investigate the ability and significance of the new soft clothing biometrics for retrieval in more challenging scenarios. One possible scenario 
could be the retrieval using newly collected query annotations describing unseen subjects' images derived from different viewpoints in which some clothing attributes can be occluded or difficult to observe. Therefore, such an analysis appears more vulnerable to subjectivity and missing information. Another future work could be to focus on learning a fully automated clothing labelling for data images and query images for retrieval purposes.

\section{References}

1. Reid D, Nixon M, Stevenage S: Soft Biometrics; Human Identification using Comparative Descriptions. IEEE Trans. PAMI. (2014)

2. Thornton J, Baran-Gale J, Butler D et al.: Person attribute search for large-area video surveillance. In: IEEE Int. Conf. on Technologies for Homeland Security (HST). (2011)

3. Adjeroh D, Deng C, Piccirilli M et al.: Predictability and correlation in human metrology. In: IEEE Int. Workshop on Information Forensics and Security (WIFS). (2010)

4. Samangooei S, Nixon MS: Performing content-based retrieval of humans using gait biometrics. Multimedia Tools and Applications 49:195-212. (2010)

5. Niinuma K, Park U, Jain AK: Soft biometric traits for continuous user authentication. IEEE Trans. on Information Forensics and Security (IFS) 5:771-780. (2010)

6. Jain AK, Park U: Facial marks: Soft biometric for face recognition. In: Proc. 16th IEEE International Conference on Image Processing (ICIP). (2009)

7. Park U, Jain AK: Face matching and retrieval using soft biometrics. IEEE Trans. on IFS 5:406-415. (2010)

8. Tome P, Fierrez J, Vera-Rodriguez R et al.: Soft Biometrics and Their Application in Person Recognition at a Distance. IEEE Trans. on IFS. (2014)

9. Vaquero DA, Feris RS, Tran D et al.: Attribute-based people search in surveillance environments. In: Workshop on Applications of Computer Vision (WACV). IEEE. (2009)

10. Bossard L, Dantone M, Leistner C et al.: Apparel classification with style. In: Computer Vision-ACCV. Springer. (2013)

11. Wang H, Bao X, Choudhury RR et al.: InSight: recognizing humans without face recognition. In: Proc. 14th Workshop on Mobile Computing Systems and Apps. (2013)

12. Zhu J, Liao S, Lei $Z$ et al.: Pedestrian Attribute Classification in Surveillance: Database and Evaluation. In: IEEE International Conference on Computer Vision (ICCV). (2013)

13. Layne R, Hospedales TM, Gong S: Person Re-identification by Attributes, In: BMVC. (2012)

14. Wang Y-F, Chang EY, Cheng, KP: A video analysis framework for soft biometry security surveillance. In: Proc. 3rd ACM Int. Workshop on Video Surv. \& Sensor Networks. (2005)

15. Gallagher AC, Chen $\mathrm{T}$ : Clothing cosegmentation for recognizing people. In: IEEE Conference on Computer Vision and Pattern Recognition (CVPR). (2008)

16. Jaha ES, Nixon MS: Soft Biometrics for Subject Identification using Clothing Attributes. In: IEEE International Joint Conference on Biometrics (IJCB). (2014)

17. Macleod MD, Frowley JN, Shepherd JW: Whole body information: Its relevance to eyewitnesses. In: Adult eyewitness testimony: Current trends and developments. (1994)

18. Shutler J, Grant M, Nixon MS et al.: On a large sequence-based human gait database. In: RASC. (2002)

19. Joachims T: Optimizing search engines using clickthrough data. In: Proc. 8th ACM SIGKDD Int. Conf. on Knowledge discovery and data mining. (2002)

20. Parikh D, Grauman K: Relative attributes. In: IEEE ICCV. (2011) 